\title{
Bianchi type-Vlo Universe with wet dark fluid
}

\author{
Raghavendra Chaubey
}

DST-Centre for Interdisciplinary Mathematical Sciences, Faculty of Science, Banaras Hindu University, Varanasi, India; rchaubey@bhu.ac.in

Received 4 February 2011; revised 18 March 2011; accepted 27 March 2011.

\section{ABSTRACT}

The Bianchi type-Vlo universe filled with dark energy from a wet dark fluid has been considered. A new equation of state for the dark energy component of the universe has been used. It is modeled on the equation of state $p=$ $\gamma\left(\rho-\rho_{*}\right)$ which can describe a liquid, for example water. The exact solutions to the corresponding field equations are obtained in quadrature form. The solution for constant deceleration parameter have been studied in detail for power-law and exponential forms both. The case $\gamma=0, \gamma=1$ and $\gamma=1 / 3$ have been also analysed.

Keywords: Cosmological Models; Wet Dark Fluid; Cosmological Parameters

\section{INTRODUCTION}

The nature of the dark energy component of the universe [1-3] remains one of the deepest mysteries of cosmology. There is certainly no lack of candidates: cosmological constant, quintessence [4-6], k-essence [7-9], phantom energy [10-12]. Modifications of the Friedmann equation such as Cardassian expansion $[13,14]$ as well as what might be derived from brane cosmology [15-17] have also been used to explain the acceleration of the universe. A particular case of the linear equation of state has used in the cosmological context by Xanthopuolos [18], he considered space-times with two hypersueface orthogonal, spacelike, commuting killing fields.

In this work, we use Wet Dark Fluid (WDF) as a model for dark energy. This model is in the spirit of the generalized Chaplygin gas (GCG) [19], where a physically motivated equation of state is offered with properties relevant for the dark energy problem. Here the motivation stems from an empirical equation of state proposed by Tait [20] and Hayword [21] to treat water and aqueous solution. The equation of state for WDF is very simple,

$$
p_{W D F}=\gamma\left(\rho_{W D F}-\rho_{*}\right)
$$

and is motivated by the fact that it is a good approximation for many fluids, including water, in which the internal attraction of the molecules makes negative pressures possible. One of the virtues of this model is that the square of the sound speed, $c_{s}{ }^{2}$, which depends on $\partial p / \partial \rho$, can be positive (as opposed to the case of phantom energy, say), while still giving rise to cosmic acceleration in the current epoch.

We treat Eq.1.1 as a phemenological equation [22]. Holman et al. [23] have shown that this model can be made consistent with the most recent SNIa data [24], the WMAP results $[25,26]$ as well as constraints coming from measurements of the matter power spectrum [27]. The parameters $\gamma$ and $\rho_{*}$ are taken to be positive and we restrict ourselves to $0 \leq \gamma \leq 1$. Note that if $c_{s}$ denotes the adiabatic sound speed in WDF, then $\gamma=c_{s}{ }^{2}$ (refer Babichev et al. [28]).

To find the WDF energy density, we use the energy conservation equation

$$
\dot{\rho}_{W D F}+3 H\left(p_{W D F}+\rho_{W D F}\right)=0
$$

From equation of state (1.1) and using $3 H=\dot{V} / V$ in above equation, we have

$$
\rho_{W D F}=\frac{\gamma}{1+\gamma} \rho_{*}+\frac{C}{V^{(1+\gamma)}}
$$

where $C$ is a constant of integration. Here $V$ is volume expansion.

WDF naturally includes two components: a piece that behaves as a cosmological constant as well as a standard fluid with an equation of state $p=\gamma \rho$. We can show that if we take $C>0$, this fluid will not violate the strong energy condition $p+\rho \geq 0$ :

$$
\begin{aligned}
p_{W D F}+\rho_{W D F} & =(1+\gamma) \rho_{W D F}-\gamma \rho_{*} \\
& =(1+\gamma) \frac{C}{V^{(1+\gamma)}} \geq 0
\end{aligned}
$$

Chaubey and Chaubey et al. ([29,30]) have studied 
Bianchi type-I and $\mathrm{V}$ universes with wet dark fluid. In this paper we study the Bianchi type-VIo universe with matter term with dark energy treated as a Dark Fluid satisfying the equation of state (1.1). The solution has been obtained in the quadrature form. The models with constant deceleration parameter have been studied in detail.

\section{BASIC EQUATION}

We take Bianchi type-VIo metric in form

$$
d s^{2}=d t^{2}-a_{1}^{2} d x^{2}-a_{2}^{2} e^{-2 m^{2} x} d y^{2}-a_{3}{ }^{2} e^{2 m^{2} x} d z^{2} .
$$

where the metric functions $a_{1}, a_{2}, a_{3}$ are functions of $t$ only and $m$ is a constant

The Einstein field equations for the metric (2.1) are written in the form

$$
\begin{aligned}
& \frac{\ddot{a}_{2}}{a_{2}}+\frac{\ddot{a}_{3}}{a_{3}}+\frac{\dot{a}_{2} \dot{a}_{3}}{a_{2} a_{3}}+\frac{m^{4}}{a_{1}^{2}}=\kappa T_{1}^{1} . \\
& \frac{\ddot{a}_{1}}{a_{1}}+\frac{\ddot{a}_{3}}{a_{3}}+\frac{\dot{a}_{1} \dot{a}_{3}}{a_{1} a_{3}}-\frac{m^{4}}{a_{1}^{2}}=\kappa T_{2}^{2} . \\
& \frac{\ddot{a}_{1}}{a_{1}}+\frac{\ddot{a}_{2}}{a_{2}}+\frac{\dot{a}_{1} \dot{a}_{2}}{a_{1} a_{2}}-\frac{m^{4}}{a_{1}^{2}}=\kappa T_{3}^{3} . \\
& \frac{\dot{a}_{1} \dot{a}_{2}}{a_{1} a_{2}}+\frac{\dot{a}_{2} \dot{a}_{3}}{a_{2} a_{3}}+\frac{\dot{a}_{3} \dot{a}_{1}}{a_{3} a_{1}}-\frac{m^{4}}{a_{1}^{2}}=\kappa T_{0}^{0} . \\
& \frac{\dot{a}_{3}}{a_{3}}-\frac{\dot{a}_{2}}{a_{2}}=0
\end{aligned}
$$

Here $\kappa$ is the gravitational constant and overhead dot denotes differentiation with respect to $t$.

The energy-momentum tensor of the source is given by

$$
T_{i}^{j}=\left(\rho_{W D F}+p_{W D F}\right) u_{i} u^{j}-p_{W D F} \delta_{i}^{j} .
$$

where $u^{i}$ is the flow vector satisfying

$$
g_{i j} u^{i} u^{j}=1 \text {. }
$$

In a co-moving system of coordinates, from Eq.2.7 we find

$$
T_{0}^{0}=\rho_{W D F}, \quad T_{1}^{1}=T_{2}^{2}=T_{3}^{3}=-p_{W D F} .
$$

Now using Eq.2.9 in Eqs.2.2-2.6 we obtain

$$
\begin{aligned}
& \frac{\ddot{a}_{2}}{a_{2}}+\frac{\ddot{a}_{3}}{a_{3}}+\frac{\dot{a}_{2} \dot{a}_{3}}{a_{2} a_{3}}+\frac{m^{4}}{a_{1}^{2}}=-\kappa p_{W D F} . \\
& \frac{\ddot{a}_{1}}{a_{1}}+\frac{\ddot{a}_{3}}{a_{3}}+\frac{\dot{a}_{1} \dot{a}_{3}}{a_{1} a_{3}}-\frac{m^{4}}{a_{1}^{2}}=-\kappa p_{W D F} . \\
& \frac{\ddot{a}_{1}}{a_{1}}+\frac{\ddot{a}_{2}}{a_{2}}+\frac{\dot{a}_{1} \dot{a}_{2}}{a_{1} a_{2}}-\frac{m^{4}}{a_{1}^{2}}=-\kappa p_{W D F} .
\end{aligned}
$$

$$
\begin{aligned}
& \frac{\dot{a}_{1} \dot{a}_{2}}{a_{1} a_{2}}+\frac{\dot{a}_{2} \dot{a}_{3}}{a_{2} a_{3}}+\frac{\dot{a}_{3} \dot{a}_{1}}{a_{3} a_{1}}-\frac{m^{4}}{a_{1}^{2}}=\kappa \rho_{W D F} . \\
& \frac{\dot{a}_{3}}{a_{3}}-\frac{\dot{a}_{2}}{a_{2}}=0
\end{aligned}
$$

From Eq.2.14 we get

$$
a_{2}=a_{3}
$$

Let $V$ be a function of $t$ defined by

$$
V=a_{1} a_{2} a_{3} .
$$

From Eqs.2.15 and 2.16, we get

$$
V=a_{1} a_{2}^{2}
$$

Now adding Eqs.2.10-2.12 and three times Eq.2.13, we get

$$
\frac{\ddot{a}_{1}}{a_{1}}+2 \frac{\ddot{a}_{2}}{a_{2}}+2\left(\frac{\dot{a}_{2}^{2}}{a_{2}^{2}}+2 \frac{\dot{a}_{1} \dot{a}_{2}}{a_{1} a_{2}}\right)-\frac{2 m^{4}}{a_{1}^{2}}=\frac{3 \kappa}{2}\left(\rho_{W D F}-p_{W D F}\right)
$$

From Eqs.2.17 and 2.18 we have

$$
\frac{\ddot{V}}{V}-\frac{2 m^{4}}{a_{1}^{2}}=\frac{3 \kappa}{2}\left(\rho_{W D F}-p_{W D F}\right) .
$$

The conservational law for the energy-momentum tensor gives

$$
\dot{\rho}_{W D F}=-\frac{\dot{V}}{V}\left(\rho_{W D F}+p_{W D F}\right) .
$$

Case 1: When $a_{1}=\sqrt{V}$

Then Eq.2.19 reduces to

$$
\frac{\ddot{V}}{V}-\frac{2 m^{4}}{V}=\frac{3 \kappa}{2}\left(\rho_{W D F}-p_{W D F}\right) .
$$

From Eqs.2.20 and 2.21 we have

$$
\dot{V}= \pm \sqrt{C_{1}+3 \kappa \rho_{W D F} V^{2}+4 m^{4} V}
$$

with $C_{1}$ being an integration constant.

Rewriting Eq.2.20 in the form

$$
\frac{\dot{\rho}}{\rho_{W D F}+p_{W D F}}=-\frac{\dot{V}}{V}
$$

and taking into account that the pressure and the energy density obeying an equation of state of type $p_{W D F}=$ $f\left(\rho_{W D F}\right)$, we conclude that $\rho_{W D F}$ and $p_{W D F}$, hence the right hand side of the Eq.2.19 is a function of $V$ only.

$$
\ddot{V}=\frac{3 \kappa}{2}\left(\rho_{W D F}-p_{W D F}\right) V+2 m^{4} \equiv F(V) .
$$

From the mechanical point of view Eq.2.24 can be interpreted as equation of motion of a single particle 
with unit mass under the force $F(V)$. Then

$$
\dot{V}=\sqrt{2[\varepsilon-U(V)]} .
$$

Here $\varepsilon$ can be viewed as energy and $U(V)$ as the potential of the force $F$. Compairing the Eqs.2.22 and 2.25 we find $\varepsilon=C_{1} / 2$ and

$$
U(V)=-\left[\frac{3}{2} \kappa \rho_{W D F} V^{2}+4 m^{4} V\right] .
$$

Finally, we write the solution to the Eq.2.22 in quadrature form

$$
\int \frac{\mathrm{d} V}{\sqrt{C_{1}+3 \kappa \rho_{W D F} V^{2}+4 m^{4} V}}=t+t_{0} .
$$

where the integration constant $t_{0}$ can be taken to be zero, since it only gives a shift in time.

From Eqs.1.3 and 2.27 we obtain

$$
\int \frac{\mathrm{d} V}{\sqrt{\frac{3 \kappa \gamma}{1+\gamma} \rho_{*} V^{2}+3 \kappa C V^{(1-\gamma)}+4 m^{4} V+C_{1}}}=t+t_{0} .
$$

Case 2: When $a_{2}=\sqrt{V}$

Then Eq.2.19 reduces to

$$
\frac{\ddot{V}}{V}-2 m^{4}=\frac{3 \kappa}{2}\left(\rho_{W D F}-p_{W D F}\right) .
$$

From Eqs.2.20 and 2.29 we have

$$
\dot{V}= \pm \sqrt{C_{1}+\left(3 \kappa \rho_{W D F}+4 m^{4}\right) V^{2}}
$$

with $C_{1}$ being an integration constant.

From Eq.2.23 and taking into account that the pressure and the energy density obeying an equation of state of type $p_{W D F}=f\left(\rho_{W D F}\right)$, we conclude that $\rho_{W D F}$ and $p_{W D F}$, hence the right hand side of the Eq.2.19 is a function of $V$ only.

$$
\ddot{V}=\frac{3 \kappa}{2}\left(\rho_{W D F}-p_{W D F}\right) V+2 m^{4} V \equiv F(V) .
$$

From the mechanical point of view Eq.2.31 can be interpreted as equation of motion of a single particle with unit mass under the force $F(V)$. Then

$$
\dot{V}=\sqrt{2[\varepsilon-U(V)]} .
$$

Here $\varepsilon$ can be viewed as energy and $U(V)$ as the potential of the force $F$. Compairing the Eqs.2.30 and 2.32 we find $\varepsilon=C_{1} / 2$ and

$$
U(V)=-\left[\frac{3}{2} \kappa \rho_{W D F}+4 m^{4}\right] V^{2} .
$$

Finally, we write the solution to the Eq.2.30 in quadrature form

$$
\int \frac{\mathrm{d} V}{\sqrt{C_{1}+\left(3 \kappa \rho_{W D F}+4 m^{4}\right) V^{2}}}=t+t_{0} .
$$

where the integration constant $t_{0}$ can be taken to be zero, since it only gives a shift in time.

From Eqs.1.3 and 2.34 we obtain

$$
\int \frac{\mathrm{d} V}{\sqrt{\left(\frac{3 \kappa \gamma}{1+\gamma} \rho_{*}+4 m^{4}\right) V^{2}+3 \kappa C V^{(1-\gamma)}+C_{1}}}=t+t_{0} .
$$

\section{SOME PARTICULAR CASES}

Case 1: When $a_{1}=\sqrt{V}$

Case I. $\gamma=0$ (Dust Universe)

Eq.2.28 reduces to

$$
\int \frac{\mathrm{d} V}{\sqrt{\left(\frac{3}{2} \kappa C+4 m^{4}\right) V+C_{1}}}=t
$$

which gives

$$
V=\frac{\left(\frac{3 \kappa C}{4}+2 m^{4}\right)^{2} t^{2}-C_{1}}{\left(\frac{3 \kappa C}{2}+4 m^{4}\right)}
$$

From Eqs.2.15, 2.17 and 3.2, we get

$$
\begin{aligned}
& a_{1}(t)=\left[\frac{\left(\frac{3 \kappa C}{4}+2 m^{4}\right)^{2} t^{2}-C_{1}}{\left(\frac{3 \kappa C}{2}+4 m^{4}\right)}\right]^{1 / 2} \\
& a_{2}(t)=a_{3}(t)=\left[\frac{\left(\frac{3 \kappa C}{4}+2 m^{4}\right)^{2} t^{2}-C_{1}}{\left(\frac{3 \kappa C}{2}+4 m^{4}\right)}\right]^{1 / 4}
\end{aligned}
$$

From Eqs.1.3 and 3.2 we have

$$
\rho_{W D F}=C\left[\frac{\left(\frac{3 \kappa C}{4}+2 m^{4}\right)^{2} t^{2}-C_{1}}{\left(\frac{3 \kappa C}{2}+4 m^{4}\right)}\right]^{-1}
$$

and from Eqs.1.1 and 3.5 we get

$$
p_{W D F}=0
$$

The physical quantities of observational interest in cosmology are the expansion scalar $\theta$, the mean anisotropy parameter $A$, the shear scalar $\sigma^{2}$ and the deceleration parameter $q$. They are defined as [31,32] 


$$
\begin{gathered}
\theta=3 H . \\
A=\frac{1}{3} \sum_{i=1}^{3}\left(\frac{\Delta H_{i}}{H}\right)^{2} . \\
\sigma^{2}=\frac{1}{2}\left(\sum_{i=1}^{3} H_{i}^{2}-3 H^{2}\right)=\frac{3}{2} A H^{2} . \\
q=\frac{\mathrm{d}}{\mathrm{d} t}\left(\frac{1}{H}\right)-1 .
\end{gathered}
$$

with the use of Eqs.3.7-3.10 we can express the physical quantities as

$$
\begin{gathered}
\theta=\frac{2\left(\frac{3 \kappa C}{4}+2 m^{4}\right)^{2} t}{\left(\frac{3 \kappa C}{4}+2 m^{4}\right)^{2} t^{2}-C_{1}} \\
\left.\sigma^{2}=\frac{1}{12}\left[\frac{\left(\frac{3}{8}\right.}{\left(\frac{3 \kappa C}{4}+2 m^{4}\right)^{2} t}\right]^{2}\right]^{2} t^{2}-C_{1} \\
q=\frac{1}{2}+\frac{\left(3 m^{4} C\right.}{\left(\frac{3 \kappa}{4}+2 m^{4}\right)^{2} t^{2}}
\end{gathered}
$$

For large cosmic time, the shear dies out.

Case II. $\gamma=1$ (Zeldovich Fluid)

Eq.2.28 reduces to

$$
\int \frac{\mathrm{d} V}{\sqrt{\frac{3}{4} \rho_{*} V^{2}+4 m^{4} V+\left(\frac{3}{2} \kappa C+C_{1}\right)}}=t
$$

which gives

$$
V=\frac{\sqrt{6 \rho_{*}\left(3 \kappa C+2 C_{1}\right)-64 m^{8}}}{3 \rho_{*}} \sinh \left(\frac{\sqrt{3} \rho_{*}}{2} t\right)-\frac{8 m^{4}}{3 \rho_{*}}
$$

when $\rho_{*}>\frac{32 m^{8}}{3\left(3 \kappa C+2 C_{1}\right)}$

$$
V=\left(\frac{2}{\sqrt{3 \rho_{*}}} e^{\frac{\sqrt{3 \rho_{*}}}{2} t}-\frac{8 m^{4}}{3 \rho_{*}}\right)
$$

when $\rho_{*}=\frac{32 m^{8}}{3\left(3 \kappa C+2 C_{1}\right)}$

$$
V=\frac{\sqrt{64 m^{8}-6 \rho_{*}\left(3 \kappa C+2 C_{1}\right)}}{3 \rho_{*}} \cosh \left(\frac{\sqrt{3} \rho_{*}}{2} t\right)-\frac{8 m^{4}}{3 \rho_{*}}
$$

when $\rho_{*}<\frac{32 m^{8}}{3\left(3 \kappa C+2 C_{1}\right)}$

We consider these subcases separately.

Case II(a) $\rho_{*}=\frac{32 m^{8}}{3\left(3 \kappa C+2 C_{1}\right)}$

Then

$$
\begin{aligned}
& a_{1}(t)=\left(\frac{2}{\sqrt{3 \rho_{*}}} e^{\frac{\sqrt{3 \rho_{*}}}{2} t}-\frac{8 m^{4}}{3 \rho_{*}}\right)^{1 / 2} \\
& a_{2}(t)=a_{3}(t)=\left(\frac{2}{\sqrt{3 \rho_{*}}} e^{\frac{\sqrt{3 \rho_{*}}}{2} t}-\frac{8 m^{4}}{3 \rho_{*}}\right)^{1 / 4}
\end{aligned}
$$

From Eqs.1.3 and 3.17, we have

$$
\rho_{W D F}=\frac{\rho_{*}}{2}+C\left(\frac{2}{\sqrt{3 \rho_{*}}} e^{\frac{\sqrt{3 \rho_{*}}}{2} t}-\frac{8 m^{4}}{3 \rho_{*}}\right)^{-2}
$$

and from Eqs.1.1 and 3.21, we get

$$
p_{W D F}=-\frac{\rho_{*}}{2}+C\left(\frac{2}{\sqrt{3 \rho_{*}}} e^{\frac{\sqrt{3 \rho_{*}}}{2} t}-\frac{8 m^{4}}{3 \rho_{*}}\right)^{-2}
$$

with the use of Eqs.3.7-3.10 we can express the physical quantities as

$$
\begin{aligned}
& \theta=\frac{\frac{\sqrt{3 \rho_{*}}}{2} e^{\frac{\sqrt{3 \rho_{*}}}{2} t}}{e^{\frac{\sqrt{3 \rho_{*}}}{2} t}-\frac{4 m^{4}}{\sqrt{3 \rho_{*}}}} \\
& A=\frac{1}{8}
\end{aligned}
$$

The model has no singularity.

Case II(b) $\rho_{*}>\frac{32 m^{8}}{3\left(3 \kappa C+2 C_{1}\right)}$

Then for small $t$ (i.e. near singularity $t=0$ ), 


$$
\sinh \left(\frac{\sqrt{3 \rho_{*}}}{2} t\right) \approx \frac{\sqrt{3 \rho_{*}}}{2} t
$$

Then Eq.3.16 reduces to

$$
V=\sqrt{\frac{\left(3 \kappa C+2 C_{1}\right)}{2}-\frac{16 m^{8}}{3 \rho_{*}}} t-\frac{8 m^{4}}{3 \rho_{*}}
$$

Then

$$
\begin{aligned}
& a_{1}(t)=\left[\sqrt{\frac{\left(3 \kappa C+2 C_{1}\right)}{2}-\frac{16 m^{8}}{3 \rho_{*}}} t-\frac{8 m^{4}}{3 \rho_{*}}\right]^{1 / 2} \\
& a_{2}(t)=a_{3}(t)=\left[\sqrt{\frac{\left(3 \kappa C+2 C_{1}\right)}{2}}-\frac{16 m^{8}}{3 \rho_{*}} t-\frac{8 m^{4}}{3 \rho_{*}}\right]^{1 / 4}
\end{aligned}
$$

From Eqs.1.3 and 3.28, we have

$$
\rho_{W D F}=\frac{\rho_{*}}{2}+C\left[\sqrt{\frac{\left(3 \kappa C+2 C_{1}\right)}{2}-\frac{16 m^{8}}{3 \rho_{*}}} t-\frac{8 m^{4}}{3 \rho_{*}}\right]^{-2}
$$

and from Eqs.1.1 and 3.21, we get

$$
p_{W D F}=-\frac{\rho_{*}}{2}+C\left[\sqrt{\frac{\left(3 \kappa C+2 C_{1}\right)}{2}-\frac{16 m^{8}}{3 \rho_{*}}} t-\frac{8 m^{4}}{3 \rho_{*}}\right]^{-2}
$$

with the use of Eqs.3.7-3.10 we can express the physical quantities as

$$
\left.\begin{array}{c}
\theta=\frac{\sqrt{\frac{\left(3 \kappa C+2 C_{1}\right)}{2}-\frac{16 m^{8}}{3 \rho_{*}}}}{\sqrt{\frac{\left(3 \kappa C+2 C_{1}\right)}{2}-\frac{16 m^{8}}{3 \rho_{*}} t-\frac{8 m^{4}}{3 \rho_{*}}}} \\
\sigma^{2}=\frac{1}{48}\left[\frac{\frac{1}{8}}{\sqrt{\frac{\left(3 \kappa C+2 C_{1}\right)}{2}-\frac{16 m^{8}}{3 \rho_{*}}}}\right]^{2} \\
q=2
\end{array}\right]
$$

The model has no singularity.

Case II(c) $\rho_{*}<32 m^{8} /\left(3\left(3 \kappa C+2 C_{1}\right)\right)$
Then for small $t$ (i.e. near singularity $t=0$ ),

$$
\cosh \left(\frac{\sqrt{3 \rho_{*}}}{2} t\right) \approx 1+\frac{3 \rho_{*}}{4} t^{2}
$$

Then Eq.3.18 reduces to

$$
\begin{aligned}
V= & \sqrt{4 m^{8}-\frac{3}{8} \rho_{*}\left(3 \kappa C+2 C_{1}\right) t^{2}} \\
& +\frac{\sqrt{64 m^{8}-6 \rho_{*}\left(3 \kappa C+2 C_{1}\right)}-8 m^{4}}{3 \rho_{*}}
\end{aligned}
$$

Then

$$
\begin{aligned}
& a_{1}(t)= {\left[\sqrt{4 m^{8}-\frac{3}{8} \rho_{*}\left(3 \kappa C+2 C_{1}\right)} t^{2}\right.} \\
&+\left.\frac{\sqrt{64 m^{8}-6 \rho_{*}\left(3 \kappa C+2 C_{1}\right)}-8 m^{4}}{3 \rho_{*}}\right]^{1 / 2} \\
& a_{2}(t)=a_{3}(t)=\left[\sqrt{4 m^{8}-\frac{3}{8} \rho_{*}\left(3 \kappa C+2 C_{1}\right)} t^{2}\right. \\
&\left.+\frac{\sqrt{64 m^{8}-6 \rho_{*}\left(3 \kappa C+2 C_{1}\right)}-8 m^{4}}{3 \rho_{*}}\right]^{1 / 4}
\end{aligned}
$$

From Eqs.1.3 and 3.38, we have

$$
\begin{aligned}
\rho_{W D F}= & \frac{\rho_{*}}{2}+C\left[\sqrt{4 m^{8}-\frac{3}{8} \rho_{*}\left(3 \kappa C+2 C_{1}\right)} t^{2}\right. \\
& \left.+\frac{\sqrt{64 m^{8}-6 \rho_{*}\left(3 \kappa C+2 C_{1}\right)}-8 m^{4}}{3 \rho_{*}}\right]^{-2}
\end{aligned}
$$

and from Eqs.1.1 and 3.41, we get

$$
\begin{aligned}
p_{W D F}= & -\frac{\rho_{*}}{2}+C\left[\sqrt{4 m^{8}-\frac{3}{8} \rho_{*}\left(3 \kappa C+2 C_{1}\right)} t^{2}\right. \\
& \left.+\frac{\sqrt{64 m^{8}-6 \rho_{*}\left(3 \kappa C+2 C_{1}\right)}-8 m^{4}}{3 \rho_{*}}\right]^{-2}
\end{aligned}
$$

with the use of Eqs.3.7-3.10 we can express the physical quantities as

$$
A=\frac{1}{8}
$$

$$
\theta=\frac{\sqrt{16 m^{8}-\frac{3}{2} \rho_{*}\left(3 \kappa C+2 C_{1}\right) t}}{\sqrt{4 m^{8}-\frac{3}{8} \rho_{*}\left(3 \kappa C+2 C_{1}\right)} t^{2}+\frac{\sqrt{64 m^{8}-6 \rho_{*}\left(3 \kappa C+2 C_{1}\right)}-8 m^{4}}{3 \rho_{*}}}
$$




$$
\begin{aligned}
& \sigma^{2}=\frac{1}{48}\left[\frac{\sqrt{16 m^{8}-\frac{3}{2} \rho_{*}\left(3 \kappa C+2 C_{1}\right) t}}{\sqrt{4 m^{8}-\frac{3}{8} \rho_{*}\left(3 \kappa C+2 C_{1}\right)} t^{2}+\frac{\sqrt{64 m^{8}-6 \rho_{*}\left(3 \kappa C+2 C_{1}\right)}-8 m^{4}}{3 \rho_{*}}}\right]^{2} \\
& q=\frac{1}{2}-\frac{\sqrt{64 m^{8}-6 \rho_{*}\left(3 \kappa C+2 C_{1}\right)}-8 m^{4}}{\sqrt{16 m^{8}-\frac{3}{2} \rho_{*}\left(3 \kappa C+2 C_{1}\right) t^{2}}} \\
& A=\frac{1}{2} \\
& \sigma^{2}=\frac{m^{4} e^{4 m^{2} t}}{3\left(e^{2 m^{2} t}-\frac{3 \kappa C}{8 m^{4}}\right)^{2}} \\
& q=\frac{9 \kappa C}{8 m^{4}} e^{-2 m^{2} t}-1
\end{aligned}
$$

$$
\int \frac{\mathrm{d} V}{\sqrt{4 m^{4} V^{2}+3 \kappa C V+C_{1}}}=t
$$

which gives

$$
V=\frac{1}{2 m^{2}} \sqrt{C_{1}-\frac{9 \kappa^{2} C^{2}}{16 m^{4}}} \sinh \left(2 m^{2} t\right)-\frac{3 \kappa C}{8 m^{4}}
$$

when $C_{1}>\frac{9 \kappa^{2} C^{2}}{16 m^{4}}$

$$
\begin{gathered}
V=\left(e^{2 m^{2} t}-\frac{3 \kappa C}{8 m^{4}}\right), \text { where } C_{1}=\frac{9 \kappa^{2} C^{2}}{16 m^{4}} \\
V=\frac{1}{2 m^{2}} \sqrt{\frac{9 \kappa^{2} C^{2}}{16 m^{4}}-C_{1}} \cosh \left(2 m^{2} t\right)-\frac{3 \kappa C}{8 m^{4}}
\end{gathered}
$$

where $C_{1}<\frac{9 \kappa^{2} C^{2}}{16 m^{4}}$

We consider these subcases separately.

Case I(a) when $C_{1}=\frac{9 \kappa^{2} C^{2}}{16 m^{4}}$

From Eqs.2.15, 2.17 and 3.49, we get

$$
\begin{gathered}
a_{1}(t)=1 \\
a_{2}(t)=a_{3}(t)=\sqrt{\left(e^{2 m^{2} t}-\frac{3 \kappa C}{8 m^{4}}\right)}
\end{gathered}
$$

From Eqs.1.3 and 3.49 we have

$$
\rho_{W D F}=C\left(e^{2 m^{2} t}-\frac{3 \kappa C}{8 m^{4}}\right)^{-1}
$$

and from Eqs.1.1 and 3.53 we get

$$
p_{W D F}=0
$$

with the use of Eq.3.7-3.10 we can express the physical quantities as

$$
\theta=\frac{2 m^{2} e^{2 m^{2} t}}{\left(e^{2 m^{2} t}-\frac{3 \kappa C}{8 m^{4}}\right)}
$$

For large $t$, the shear dies out.

Case I(b) when $C_{1}>\left(9 \kappa^{2} C^{2}\right) /\left(16 m^{4}\right)$

Then for small $t$ (i.e. near singularity $t=0$ ),

$$
\sinh \left(2 m^{2} t\right) \approx 2 m^{2} t
$$

Then Eq.3.48 reduces to

$$
V=\sqrt{C_{1}-\frac{9 \kappa^{2} C^{2}}{16 m^{4}}} t-\frac{3 \kappa C}{8 m^{4}}
$$

From Eqs.2.15, 2.17 and 3.60, we get

$$
\begin{gathered}
a_{1}(t)=1 \\
a_{2}(t)=a_{3}(t)=\left[\sqrt{C_{1}-\frac{9 \kappa^{2} C^{2}}{16 m^{4}} t} t-\frac{3 \kappa C}{8 m^{4}}\right]^{1 / 2}
\end{gathered}
$$

From Eqs.1.3 and 3.60 we have

$$
\rho_{W D F}=C\left[\sqrt{C_{1}-\frac{9 \kappa^{2} C^{2}}{16 m^{4}}} t-\frac{3 \kappa C}{8 m^{4}}\right]^{-1}
$$

and from Eqs.1.1 and 3.63 we get

$$
p_{W D F}=0
$$

with the use of Eqs.3.7-3.10 we can express the physical quantities as

$$
\begin{gathered}
\theta=\frac{\sqrt{C_{1}-\frac{9 \kappa^{2} C^{2}}{16 m^{4}}}}{\sqrt{C_{1}-\frac{9 \kappa^{2} C^{2}}{16 m^{4}} t-\frac{3 \kappa C}{8 m^{4}}}} \\
A=\frac{1}{2} \\
\sigma^{2}=\frac{C_{1}-\frac{9 \kappa^{2} C^{2}}{16 m^{4}}}{12\left[\sqrt{C_{1}-\frac{9 \kappa^{2} C^{2}}{16 m^{4}}} t-\frac{3 \kappa C}{8 m^{4}}\right]^{2}}
\end{gathered}
$$




$$
q=2
$$

For large $t$, the shear dies out.

Case I(c) when $C_{1}<\frac{9 \kappa^{2} C^{2}}{16 m^{4}}$

Then for small $t$ (i.e. near singularity $t=0$ ),

$$
\cosh \left(2 m^{2} t\right) \approx 1+4 m^{4} t^{2}
$$

Then Eq.3.50 reduces to

$V=2\left(\sqrt{\frac{9 \kappa^{2} C^{2}}{16 m^{4}}-C_{1}}\right) t^{2}+\frac{1}{2 m^{2}} \sqrt{\frac{9 \kappa^{2} C^{2}}{16 m^{4}}-C_{1}}-\frac{3 \kappa C}{8 m^{4}}$

From Eqs.2.15, 2.17 and 3.70, we get

$$
a_{1}(t)=1
$$

$$
a_{2}(t)
$$

$=a_{3}(t)$

$=\left[2\left(\sqrt{\frac{9 \kappa^{2} C^{2}}{16 m^{4}}-C_{1}}\right) t^{2}+\frac{1}{2 m^{2}} \sqrt{\frac{9 \kappa^{2} C^{2}}{16 m^{4}}-C_{1}}-\frac{3 \kappa C}{8 m^{4}}\right]^{1 / 2}$

From Eqs.1.3 and 3.70 we have

$$
\begin{aligned}
& \rho_{W D F} \\
= & C\left[2\left(\sqrt{\frac{9 \kappa^{2} C^{2}}{16 m^{4}}-C_{1}}\right) t^{2}+\frac{1}{2 m^{2}} \sqrt{\frac{9 \kappa^{2} C^{2}}{16 m^{4}}-C_{1}}-\frac{3 \kappa C}{8 m^{4}}\right]^{-1}
\end{aligned}
$$

and from Eqs.1.1 and 3.73 we get

$$
p_{W D F}=0
$$

with the use of Eqs.3.7-3.10 we can express the physical quantities as

$$
\begin{gathered}
\theta=\frac{4 \sqrt{\frac{9 \kappa^{2} C^{2}}{16 m^{4}}-C_{1} t}}{2\left(\sqrt{\frac{9 \kappa^{2} C^{2}}{16 m^{4}}-C_{1}}\right) t^{2}+\frac{1}{2 m^{2}} \sqrt{\frac{9 \kappa^{2} C^{2}}{16 m^{4}}-C_{1}}-\frac{3 \kappa C}{8 m^{4}}} \\
\sigma^{2}=\frac{A=\frac{1}{2}}{\left[\frac{3 \kappa^{2} C^{2}}{4 m^{4}}-\frac{4 C_{1}}{3}\right) t^{2}} \\
\left.2\left(\sqrt{\frac{9 \kappa^{2} C^{2}}{16 m^{4}}-C_{1}}\right) t^{2}+\frac{1}{2 m^{2}} \sqrt{\frac{9 \kappa^{2} C^{2}}{16 m^{4}}-C_{1}}-\frac{3 \kappa C}{8 m^{4}}\right]^{2}
\end{gathered}
$$

$$
q=\frac{1}{2}-\frac{\frac{3}{2 m^{2}} \sqrt{\frac{9 \kappa^{2} C^{2}}{16 m^{4}}-C_{1}}-\frac{9 \kappa C}{8 m^{4}}}{4\left(\sqrt{\frac{9 \kappa^{2} C^{2}}{16 m^{4}}-C_{1}}\right) t^{2}}
$$

For large $t$, the shear dies out. Case II $\gamma=1$ (Zeldovich Fluid)

Eq.2.35 reduces to

$$
\int \frac{\mathrm{d} V}{\sqrt{\left(\frac{3 \kappa}{2} \rho_{*}+4 m^{4}\right) V^{2}+3 \kappa C+C_{1}}}=t
$$

which gives

$$
V=\sqrt{\frac{3 \kappa C+C_{1}}{\frac{3 \kappa}{2} \rho_{*}+4 m^{4}}} \sinh \left(\sqrt{\frac{3 \kappa}{2} \rho_{*}+4 m^{4}}\right) t
$$

Then for small $t$ (i.e. near singularity $t=0$ ),

$$
\sinh \left(\sqrt{\frac{3 \kappa}{2} \rho_{*}+4 m^{4}}\right) t \approx\left(\sqrt{\frac{3 \kappa}{2} \rho_{*}+4 m^{4}}\right) t
$$

Then Eq.3.80 reduces to

$$
V=\left(\sqrt{3 \kappa C+C_{1}}\right) t
$$

From Eqs.2.15, 2.17 and 3.82, we get

$$
\begin{gathered}
a_{1}(t)=1 \\
a_{2}(t)=a_{3}(t)=\left[\left(\sqrt{3 \kappa C+C_{1}}\right) t\right]^{1 / 2}
\end{gathered}
$$

From Eqs.1.3 and 3.82 we have

$$
\rho_{W D F}=C\left[\left(\sqrt{3 \kappa C+C_{1}}\right) t\right]^{-1}
$$

and from Eqs.1.1 and 3.85 we get

$$
p_{W D F}=0
$$

with the use of Eqs.3.7-3.10 we can express the physical quantities as

$$
\begin{aligned}
& \theta=\frac{1}{t} \\
& A=\frac{1}{2} \\
& \sigma^{2}=\frac{1}{12 t^{2}} \\
& q=2
\end{aligned}
$$

For large cosmic time, the shear dies out and $\rho, p \rightarrow 0$ and the model reduces to vacuum.

Case III. $\gamma=\frac{1}{3}$ (Radiation) 
For $C_{1}=0, \mathbf{E q . 2 . 3 5}$ reduces to

$$
\int \frac{\mathrm{d} V}{\sqrt{\left(\frac{3 \kappa}{4} \rho_{*}+4 m^{4}\right) V^{2}+3 \kappa C V^{2 / 3}}}=t
$$

which gives

$$
V=\left[\sqrt{\frac{12 \kappa C}{3 \kappa \rho_{*}+16 m^{4}}} \sinh \left(\frac{\sqrt{3 \kappa \rho_{*}+16 m^{4}}}{3}\right) t\right]^{3 / 2}
$$

Then for small $t$ (i.e. near singularity $t=0$ ),

$$
\sinh \left(\frac{\sqrt{3 \kappa \rho_{*}+16 m^{4}}}{3}\right) t \approx \frac{\sqrt{3 \kappa \rho_{*}+16 m^{4}}}{3} t
$$

Then Eq.3.92 reduces to

$$
V=\left[\frac{2 \sqrt{3 \kappa C}}{3} t\right]^{3 / 2}
$$

From Eqs.2.15, 2.17 and 3.94, we get

$$
\begin{gathered}
a_{1}(t)=1 \\
a_{2}(t)=a_{3}(t)=\left[\frac{2 \sqrt{3 \kappa C}}{3} t\right]^{3 / 4}
\end{gathered}
$$

From Eqs.1.3 and 3.94 we have

$$
\rho_{W D F}=C\left[\frac{2 \sqrt{3 \kappa C}}{3} t\right]^{-3 / 2}
$$

and from Eqs.1.1 and 3.97 we get

$$
p_{W D F}=0
$$

with the use of Eqs.3.7-3.10 we can express the physical quantities as

$$
\begin{aligned}
\theta & =\frac{3}{2 t} \\
A & =\frac{1}{2} \\
\sigma^{2} & =\frac{3}{16 t^{2}} \\
q & =1
\end{aligned}
$$

For large cosmic time, the shear dies out and $\rho, p$ $\rightarrow 0$ and the model reduces to vacuum.

\section{MODELS WITH CONSTANT DECELERATION PARAMETER}

Case I. Power-Law

Here we take

$$
V=a t^{b}
$$

where $a$ and $b$ are constants,

Here we discuss three interesing cases

Case I(a). When $a_{1}=\sqrt{V}$

From Eq.4.1, we get

$$
\begin{aligned}
& a_{1}(t)=a^{1 / 2} t^{b / 2} \\
& a_{2}(t)=a_{3}(t)=a^{1 / 4} t^{b / 4}
\end{aligned}
$$

From Eq.1.3 and 4.1, we have

$$
\rho_{W D F}=\frac{\gamma}{1+\gamma} \rho_{*}+\frac{C}{a^{(1+\gamma)}} t^{-(1+\gamma) b}
$$

and from Eq.1.1 and 4.4, we get

$$
p_{W D F}=\gamma\left(\frac{C}{a^{(1+\gamma)}} t^{-(1+\gamma) b}-\frac{1}{1+\gamma} \rho_{*}\right)
$$

with the use of Eqs.3.7-3.10 we can express the physical quantities as

$$
\begin{aligned}
\theta & =\frac{b}{t} \\
A & =\frac{1}{8} \\
\sigma^{2} & =\frac{1}{48} \frac{b^{2}}{t^{2}} \\
q & =\frac{3}{b}-1
\end{aligned}
$$

Case I(b). When $a_{1}=V$

From Eq.4.1, we get

$$
\begin{aligned}
& a_{1}(t)=a t^{b} \\
& a_{2}(t)=a_{3}(t)=1
\end{aligned}
$$

From Eqs.1.3 and 4.1, we have

$$
\rho_{W D F}=\frac{\gamma}{1+\gamma} \rho_{*}+\frac{C}{a^{(1+\gamma)}} t^{-(1+\gamma) b}
$$

and from (1.1) and (4.12), we get

$$
p_{W D F}=\gamma\left(\frac{C}{a^{(1+\gamma)}} t^{-(1+\gamma) b}-\frac{1}{1+\gamma} \rho_{*}\right)
$$

with the use of Eqs.3.7-3.10 we can express the physical quantities as

$$
\begin{aligned}
\theta & =\frac{b}{t} \\
A & =2 \\
\sigma^{2} & =\frac{b^{2}}{3 t^{2}} \\
q & =\frac{3}{b}-1
\end{aligned}
$$


Case I(c). When $a_{1}=V^{2}$

From Eq.4.1, we get

$$
\begin{aligned}
& a_{1}(t)=a^{2} t^{2 b} \\
& a_{2}(t)=a_{3}(t)=a^{-1 / 2} t^{-b / 2}
\end{aligned}
$$

From Eqs.1.3 and 4.1, we have

$$
\rho_{W D F}=\frac{\gamma}{1+\gamma} \rho_{*}+\frac{C}{a^{(1+\gamma)}} t^{-(1+\gamma) b}
$$

and from Eqs.1.1 and 4.20, we get

$$
p_{W D F}=\gamma\left(\frac{C}{a^{(1+\gamma)}} t^{-(1+\gamma) b}-\frac{1}{1+\gamma} \rho_{*}\right)
$$

with the use of Eqs.3.7-3.10 we can express the physical quantities as

$$
\begin{aligned}
\theta & =\frac{b}{t} \\
A & =\frac{25}{2} \\
\sigma^{2} & =\frac{25}{12} \frac{b^{2}}{t^{2}} \\
q & =\frac{3}{b}-1
\end{aligned}
$$

For large $t$, the shear dies out and model has no singularity.

Case II. Exponential-Type

Here we take

$$
V=\alpha e^{\beta t}
$$

where $\alpha$ and $\beta$ are constants.

Here we discuss three interesing cases

Case II(a) when $a_{1}=\sqrt{V}$

From Eq.4.26, we get

$$
\begin{aligned}
& a_{1}(t)=\alpha^{1 / 2} e^{\frac{\beta t}{2}} \\
& a_{2}(t)=a_{3}(t)=\alpha^{1 / 4} e^{\frac{\beta t}{4}}
\end{aligned}
$$

From Eqs.1.3 and 4.26, we have

$$
\rho_{W D F}=\frac{\gamma}{1+\gamma} \rho_{*}+\frac{C}{\alpha^{(1+\gamma)}} e^{-(1+\gamma) \beta t}
$$

and from Eqs.1.1 and 4.29, we get

$$
p_{W D F}=\gamma\left(\frac{C}{\alpha^{(1+\gamma)}} e^{-(1+\gamma) \beta t}-\frac{1}{1+\gamma} \rho_{*}\right)
$$

with the use of Eqs.3.7-3.10 we can express the physical quantities as

$$
\theta=\beta
$$

$$
\begin{aligned}
A & =\frac{1}{8} \\
\sigma^{2} & =\frac{1}{48} \beta^{2} \\
q & =-1
\end{aligned}
$$

Case II(b). When $a_{1}=V$

From Eq.4.26, we get

$$
\begin{aligned}
& a_{1}(t)=\alpha e^{\beta t} \\
& a_{2}(t)=a_{3}(t)=1
\end{aligned}
$$

From Eqs.1.3 and 4.26, we have

$$
\rho_{W D F}=\frac{\gamma}{1+\gamma} \rho_{*}+\frac{C}{\alpha^{(1+\gamma)}} e^{-(1+\gamma) \beta t}
$$

and from Eqs.1.1 and 4.37, we get

$$
p_{W D F}=\gamma\left(\frac{C}{\alpha^{(1+\gamma)}} e^{-(1+\gamma) \beta t}-\frac{1}{1+\gamma} \rho_{*}\right)
$$

with the use of Eqs.3.7-3.10 we can express the physical quantities as

$$
\begin{aligned}
\theta & =\beta \\
A & =2 \\
\sigma^{2} & =\frac{\beta^{2}}{3} \\
q & =-1
\end{aligned}
$$

Case II(c). When $a_{1}=V^{2}$

From Eq.4.26, we get

$$
\begin{aligned}
& a_{1}(t)=\alpha^{2} e^{2 \beta t} \\
& a_{2}(t)=a_{3}(t)=\alpha^{-1 / 2} e^{\frac{-\beta t}{2}}
\end{aligned}
$$

From Eqs.1.3 and 4.26, we have

$$
\rho_{W D F}=\frac{\gamma}{1+\gamma} \rho_{*}+\frac{C}{\alpha^{(1+\gamma)}} e^{-(1+\gamma) \beta t}
$$

and from Eqs.1.1 and 4.45, we get

$$
p_{W D F}=\gamma\left(\frac{C}{\alpha^{(1+\gamma)}} e^{-(1+\gamma) \beta t}-\frac{1}{1+\gamma} \rho_{*}\right)
$$

with the use of Eqs.3.7-3.10 we can express the physical quantities as

$$
\begin{aligned}
\theta & =\beta \\
A & =\frac{25}{2} \\
\sigma^{2} & =\frac{25}{12} \beta^{2}
\end{aligned}
$$




$$
q=-1
$$

The model has no singularity.

\section{CONCLUSIONS}

The Bianchi type-VIo universe has been considered for a new equation of state for the Dark Energy component of the universe (known as dark wet fluid). The solution has been obtained in quadrature form. The models with constant deceleration parameter have been discussed in detail. The behaviour of the models for large time have been analyzed.

\section{REFERENCES}

[1] Riess, A.G., et al. (1998) Observational evidence from supernovae for an accelerating universe and cosmological constant. Astronomical Journal, 116, 1009. doi:10.1086/300499

[2] Perlmutter, S., et al. (1999) Measurements of $\Omega$ and $\Lambda$ from 42 high-redshift supernovae. Astrophysical Journal, 517, 565. doi:10.1086/307221

[3] Sahni, V. (2004) Dark matter and dark energy. arXiv: astro-ph/0403324 (preprint).

[4] Ratra, B. and Peebles, P.J.E. (1988) Cosmological consequences of a rolling homogeneous scalar field. Physical Review D, 37, 3406. doi:10.1103/PhysRevD.37.3406

[5] Caldwell, R.R., Dave, R. and Steinhardt, P.J. (1998) Cosmological imprint of an energy component with general equation of state. Physical Review Letters, 80, 1582. doi:10.1103/PhysRevLett.80.1582

[6] Barreiro, T., Copeland, E.J. and Nunes, N.J. (2000) Quintessence arinsing from exponential potentials. Physical Review D, 61, 127301. doi:10.1103/PhysRevD.61.127301

[7] Armendariz-Picon, C., Damour, T. and Mukhanov, V. (2001) K-inflation. Physics Letters B, 458, 209. doi:10.1016/S0370-2693(99)00603-6

[8] Armendariz-Picon, C., Mukhanov, V. and Steinhardt, P.J. (2001) Essentials of K-essence. Physical Review D, 63, 103510. doi:10.1103/PhysRevD.63.103510

[9] Gonzalez-Diaz, P.F. (2004) K-essential phantom energy: Doomsday around the corner? Physics Letters B, 586, 1 . doi:10.1016/i.physletb.2003.12.077

[10] Caldwell, R.R. (2002) A phantom menace? cosmological consequences of dark energy component with super-negative equation of state. Physics Letters B, 545, 23. doi:10.1016/S0370-2693(02)02589-3

[11] Carroll, S.M., Hoffman, M. and Trodden, M. (2003) Can the dark energy equation-of-state parameter $\omega$ be less than -1? Physical Review D, 68, 023509. doi:10.1103/PhysRevD.68.023509

[12] Elizalde, E., Nojiri, S. and Odintsov, S.D. (2004) Latetime cosmology in a (phantom) scalar-tensor theory: Dark energy and the cosmic speed up. Physical Review D, 70 , 043539. doi:10.1103/PhysRevD.70.043539

[13] Freese, K. and Lewis, M. (2002) Cardassian expansion: A model in which the universe is flat, matter dominated, and accelerating. Physics Letters $B, \mathbf{5 4 0}, 1$. doi:10.1016/S0370-2693(02)02122-6
[14] Gondolo, P. and Freese, K. (2003) Fluid interpretation of cardassian expansion. Physical Review D, 68, 063509. doi:10.1103/PhysRevD.68.063509

[15] Deffayet, C., Dvali, G.R. and Gabadadze, G. (2002) Accelerated universe from gravity leaking to extra dimensions. Physical Review D, 65, 044023. doi:10.1103/PhysRevD.65.044023

[16] Dvali, G., Gabadadze, G. and Porrati, M. (2000) 4D gravity on a brane in 5D minkowski space. Physics Letters $B$, 485, 208. doi:10.1016/S0370-2693(00)00669-9

[17] Dvali, G. and Turner, M.S. (2003) Dark energy as a modification of the friedmann equation. arXiv: astro-ph/ 0301510 .

[18] Xanthopuolos, B.C. (1987) Perfect fluids satisfying a less than extremely relativistic equation of state. Journal of Mathematical Physics, 28, 905. doi:10.1063/1.527581

[19] Gorini, V., Kamenshchik, A., Moschella, U. and Pasquier, V. (2004) The chaplygin gas as a model for dark energy. arXiv: gr-qc/0403062 (preprint).

[20] Tait, P.G. (1888) The voyage of H.M.S. challenger. London: H.M.S.O., 2, 1-73.

[21] Hayward, A.T.J. (1967) Compressibility equations for liquids: A comprative study. British Journal of Applied Physics, 18, 965. doi:10.1088/0508-3443/18/7/312

[22] Chiba, T., Sugiyama, N. and Nakamura, T. (1998) Observational tests of X-matter models. Monthly Notices of the Royal Astronomical Society, 301, 72. doi:10.1046/j.1365-8711.1998.02012.x

[23] Holman, R. and Naidu, S. (2005) Dark energy from wet dark fluid. arXiv: astro-ph/0408102 (preprint).

[24] Riess, A., et al., (2004) Type Ia supernova discoveries at $\mathrm{z}>1$ from the hubble space telescope: Evidence for past deceleration and constraint on dark energy evolution. arXiv: astro-ph/0402512 (preprint).

[25] Bennett, C.L., et al., (2003) First-year Wilkinson Microwave Anisotropy Probe (WMAP) obsevations: Preliminary maps and basic results. Astrophysical Journal Supplement, 148, 1. doi:10.1086/345346

[26] Spergel, D.N. (2003) First-year Wilkinson Microwave Anisotropy Probe (WMAP) obsevations: Determination of cosmological parameters. Astrophysical Journal Supplement, 148, 175. doi:10.1086/377226

[27] Tegmark, M., Hamilton, A.J.S. and Xu, Y.Z. (2002) The power spectrum of galaxies in the $2 \mathrm{dF} 100 \mathrm{k}$ redshift survey. MNRAS, 335, 887. doi:10.1046/j.1365-8711.2002.05622.x

[28] Babichev, E., Dokuchaev, V. and Eroshenkos, Y. (2004) Dark energy cosmology with generalized linear equation of state. arXiv: astro-ph/0407190 (preprint).

[29] Singh, T. and Chaubey, R. (2008) Bianchi type-I universe with wet dark fluid. Pramana-Journal of Physics, 71, 447-458.

[30] Chaubey, R. (2009) Bianchi type-V universe with wet dark fluid. Astrophysics and Space Science, 321, 241-246. doi:10.1007/s10509-009-0027-5

[31] Singh, T. and Chaubey, R. (2007) Bianchi type-V cosmological models with a viscous fluid and $\Lambda$-term. Pramana-Journal of Physics, 68, 721-734.

[32] Singh, T. and Chaubey, R. (1998) Bianchi type-V and VIo universes with dilaton and magnetic fields. International Journal of Modern Physics D, 15, 111. 